\title{
Is COVID-19 associated with latent toxoplasmosis?
}

\author{
Salman Ghaffari ${ }^{1} \cdot$ Narges Kalantari $^{2}$ (D) Tahmineh Gorgani-Firouzjaee $^{3} \cdot$ Masomeh Bayani $^{3} \cdot$ Farzaneh Jalali $^{4}$. \\ Meysam Aghajani Daroonkola ${ }^{5}$
}

Received: 9 July 2021 / Accepted: 16 October 2021 / Published online: 25 October 2021

(C) The Author(s), under exclusive licence to Springer-Verlag GmbH Germany, part of Springer Nature 2021

\begin{abstract}
The present study aimed to evaluate the possible association between coronavirus disease 2019 (COVID-19) and latent Toxoplasma gondii infection in a group of patients and healthy individuals. Blood samples were obtained from 269 PCRpositive COVID-19 patients. The serum was separated and tested for the existence of anti-T. gondii antibodies (IgG) using a commercial enzyme-linked immunosorbent assay kit. The prevalence of latent toxoplasmosis between a subgroup of the patients (aged under 55 years old) and COVID-19 negative individuals was compared. Anti-T. gondii antibodies were found in 226/269 (84.0\%) patients with COVID-19. Anti-Toxoplasma antibodies were detected in 72/91 (79.1\%) cases and 96/123 (78.0\%) COVID-19 negative individuals (odd ratio $=1.1$; 95\% confidence interval: $0.55-2.07, P=0.85$ ). The median and interquartile range (IQR) of the IgG titer were not statistically significant different between case (97.3 [31.0-133.5]) and control groups (34.4 [13.0-144.5]) $(P=0.10)$. These findings demonstrated that latent Toxoplasma infection is prevalent amongst the COVID-19 patients. It also did not find any significant association between chronic toxoplasmosis and COVID-19.
\end{abstract}

Keywords COVID-19 $\cdot$ Seroprevalence $\cdot$ T. gondii $\cdot$ Toxoplasmosis

\section{Introduction}

The severe acute respiratory syndrome coronavirus-2 (SARS-CoV-2), also known as coronavirus disease 2019 (COVID-19), started in Wuhan, China in mid-December 2019 (El-Sayed et al. 2021a; Sharma et al. 2020). The disease spread around the world rapidly, infecting approximately $235,730,857$ million people, with $4,816,146$ million

Responsible Editor: Lotfi Aleya

Narges Kalantari

nfkala@gmail.com

1 Department of Parasitology and Mycology, School of Medicine, Babol University of Medical Sciences, Babol, Iran

2 Cellular and Molecular Biology Research Centre, Health Research Institute, Babol University of Medical Sciences, Babol, Iran

3 Infectious Diseases and Tropical Medicine Research Centre, Babol University of Medical Sciences, Babol, Iran

4 Laboratory Sciences Group, Faculty of Para-Medicine, Babol University of Medical Sciences, Babol, Iran

5 Shahid Yahyanejad Hospital, Babol University of Medical Sciences, Babol, Iran deaths by 4 October 2021, as reported by https://www.world ometers.info/coronavirus/. The data obtained from multiple studies provides strong evidence confirming that certain species of bats are the natural reservoir host and source of SARS-CoV-2 (El-Sayed and Kamel 2021). However, the disease became widespread in human populations through the respiratory route, mainly by large droplets or aerosols (Mohanty et al. 2020). The clinical features of COVID19 disease vary amongst human populations. Most cases develop mild or uncomplicated illness but severe disease requiring hospitalization is detected in some patients (Hassan et al. 2020). According to literature, SARS-CoV-2 can infect several organs and prompt life-threatening clinical symptoms that can lead to cardiovascular events, severe uncontrolled immune response (cytokine storm), damage of the brain stem and/ or respiratory failure which may result in death (El-Sayed et al. 2021b).

In the first few months of the pandemic, the susceptibility of human societies appeared to be varied ranging from mild to very high morbidity. In contrast with general anticipations, wealthier communities living under better hygienic conditions seem to be more at risk than poorer societies (Jankowiak et al. 2020). The factors influencing different populations' susceptibility in this pandemic were evaluated 
by some researchers. For example, a study suggested that BCG vaccination and exposure to tuberculosis may create a non-specific protection against the COVID-19 infection (Sala and Miyakawa 2020). Jankowiak and colleagues hypothesized that common infections, which may be linked to a less hygienic lifestyle, may prompt the human immune system and therefore facilitate some protection against COVID-19. They reported that toxoplasmosis was positively related to COVID-19 delay by the linear regression model and Gross Domestic Product (GDP) (Jankowiak et al. 2020). On the other hand, a recent study reports that toxoplasmosis increased the severity of COVID-19 (Roe 2021).

Toxoplasma gondii infection is endemic in the north of Iran and the seroprevalence of latent toxoplasmosis ranges from $60.6 \%$ to $82.5 \%$ (Kalantari et al. 2018). The current study aimed to evaluate the seroprevalence of $T$. gondii infection amongst patients with COVID-19. The possible association between COVID-19 and latent $T$. gondii infection in a group of patients with COVID-19 and COVID-19 negative individuals was also assessed.

\section{Methods and materials}

\section{Study design}

Two-hundred and sixty-nine PCR-positive COVID-19 patients were enrolled in the current study. All patients were hospitalized at the Babol University of Medical Sciences affiliated hospitals, Babol, Iran, from August 2020 to February 2021 . The sample size was calculated using the formula as described below (https://library2.lincoln.ac.nz/docum ents/Sample-size.pdf):

$N=\frac{Z^{2} \times P(1-P)}{d^{2}}$

$* N, Z, P$ and $d$ indicated sample size, 1.96 , a proportion of the population having the characteristic and the degree of precision, respectively. The minimum sample size for the determination of seroprevalence rates of latent toxoplasmosis was 188 if $Z=1.96, P=60 \%, 1-P=40 \%$ and $d=0.07$ were used.

Moreover, the prevalence of latent toxoplasmosis between a subgroup of the patients ( 91 cases aged under 55 years old) and COVID-19 negative individuals (123 persons) was investigated. The data of 123 subjects without COVID-19 was obtained from our previous studies, which were performed prior to the SARS-CoV-2 pandemic. This control group was composed of 61 healthy females (Kalantari et al. 2015) and 62 blood donors aged over 29 years old (Kalantari et al. 2018).

\section{Sample collection}

A blood sample was taken from each participant, and the serum was separated after clot formation by centrifugation at $4000 \mathrm{rpm}$ for $5 \mathrm{~min}$. The sera were collected in Eppendorf tubes and then transported in an icebox to the MicrobiologyParasitology group, Para-Medical Faculty, Babol University of Medical Sciences. They were kept at $-20^{\circ} \mathrm{C}$ until tested.

\section{Sociodemographic and clinical data}

Sociodemographic data (age and gender) and clinical information such as respiratory rate, $\mathrm{O}_{2}$ saturation, cough and fever were obtained for all patients using a questionnaire form. All relevant data was obtained from the physicians and medical examination records. These variables were selected based on literature (Hassan et al. 2020).

\section{Serological analysis}

The sera were tested for the presence of IgG antibodies against $T$. gondii by a commercially available enzyme-linked immunosorbent assay (ELISA) (EUROIMMUN, Germany) according to the manufacturer's instructions. The optical density of IgG antibody titers was provided at $450 \mathrm{~nm}$ by an automatic ELISA reader. The results were interpreted based on the kit's recommendations. IgG titers of less than $8 \mathrm{IU} / \mathrm{mL}$, between 8 and $11 \mathrm{IU} / \mathrm{mL}$ and higher than $11 \mathrm{IU} /$ $\mathrm{mL}$ were considered negative, border line and positive, respectively. The sensitivity of the test was $99.9 \%$, and the specificity was $100 \%$.

\section{Statistical analysis}

SPSS v. 22.0 software (SPSS Inc., USA) was used to analyse the results, and $P<0.05$ was considered statistically significant. The association between age, gender, clinical signs of the patients and latent $T$. gondii infection was evaluated by Chi-squared test $\left(X^{2}\right)$. Odd ratios (OR) and $95 \%$ confidence intervals (CIs) were obtained using logistical regression. Age values and $\operatorname{IgG}$ titer were stated as median values and interquartile ranges (IQRs), and were compared using the Mann-Whitney $U$ test.

\section{Results}

The mean and standard deviation of the patients age were $60.9 \pm 17.6$ years, ranging from 9 to 90 years. One-hundred and five out of 269 individuals (39.0\%) were male, and 164 (61.0\%) were female. Anti-T. gondii antibodies were found in 
226/269 (84.0\%) patients with COVID-19. The frequency of IgG antibodies against $T$. gondii according to demographic and clinical data amongst patients with COVID-19 is shown in Table 1 . The prevalence of IgG antibodies against $T$. gondii had a slightly higher rate in patients aged over 60 years, in comparison with other age groups $(P=0.07)$. Statistical
Table 1 Seroprevalence of $T$. gondii infection according to the demographic and clinical data of patients with COVID19 (269) attending the Babol University affiliated Hospital, Babol, Iran

\begin{tabular}{|c|c|c|c|c|}
\hline Variable & No. tested & Seropositivity $N(\%)$ & OR $(95 \% \mathrm{CI})$ & $P$ value \\
\hline \multicolumn{5}{|l|}{ Age } \\
\hline$<40$ & 40 & $30(75)$ & & \\
\hline $40-60$ & 76 & $61(80.3)$ & $0.7(0.30-1.84)$ & $0.33^{*}$ \\
\hline$>60$ & 153 & $135(88.2)$ & $0.5(0.27-1.15)$ & 0.08 \\
\hline \multicolumn{5}{|l|}{ Gender } \\
\hline Male & 105 & $89(84.8)$ & $0.9(0.47-1.79)$ & 0.79 \\
\hline Female & 164 & $137(83.5)$ & & \\
\hline \multicolumn{5}{|c|}{ Hospital wards } \\
\hline General & 172 & $142(82.6)$ & $0.9(0.42-1.89)$ & 0.75 \\
\hline ICU & 70 & $59(84.3)$ & & \\
\hline NA & 27 & - & & \\
\hline \multicolumn{5}{|c|}{ Respiratory rate } \\
\hline$<28$ & 150 & $122(81.3)$ & $0.6(0.32-1.24)$ & 0.18 \\
\hline$>28$ & 119 & $104(87.4)$ & & \\
\hline \multicolumn{5}{|c|}{$\mathrm{O}_{2}$ saturation } \\
\hline$<93$ & 147 & $126(85.7)$ & & \\
\hline $93-95$ & 49 & $39(79.6)$ & $1.5(0.67-3.54)$ & $0.21^{*}$ \\
\hline$>95$ & 73 & $61(83.6)$ & $0.8(0.30-3.95)$ & 0.37 \\
\hline \multicolumn{5}{|l|}{ Bleeding } \\
\hline Yes & 58 & $52(89.7)$ & $1.8(0.77-4.67)$ & 019 \\
\hline No & 211 & $174(82.5)$ & & \\
\hline \multicolumn{5}{|l|}{ Trombose } \\
\hline Yes & 13 & $10(76.9)$ & $0.6(0.16-2.34)$ & 0.49 \\
\hline No & 256 & $216(84.3)$ & & \\
\hline \multicolumn{5}{|l|}{ Cough } \\
\hline Yes & 217 & $185(85.3)$ & $1.5(0.22-3.33)$ & 0.26 \\
\hline No & 52 & $41(78.8)$ & & \\
\hline \multicolumn{5}{|l|}{ Dyspnea } \\
\hline Yes & 150 & $125(83.3)$ & $0.9(0.46-1.72)$ & 0.73 \\
\hline No & 119 & $101(84.9)$ & & \\
\hline \multicolumn{5}{|l|}{ Fever } \\
\hline Yes & 221 & $187(84.6)$ & $1.3(0.56-2.86)$ & 0.57 \\
\hline No & 48 & $39(81.3)$ & & \\
\hline \multicolumn{5}{|c|}{ Gastrointestinal signs } \\
\hline Yes & 45 & $37(82.2)$ & $0.9(0.37-1.99)$ & 0.61 \\
\hline No & 225 & $189(84.0)$ & & \\
\hline \multicolumn{5}{|c|}{ Muscular Pain } \\
\hline Yes & 151 & $129(85.4)$ & $1.6(0.80-3.12)$ & 0.10 \\
\hline No & 85 & $66(77.6)$ & & \\
\hline NA & 34 & - & & \\
\hline \multicolumn{5}{|l|}{ Headache } \\
\hline Yes & 156 & $132(84.6)$ & $1.4(0.70-2.86)$ & 0.18 \\
\hline No & 78 & $62(79.5)$ & & \\
\hline NA & 35 & - & & \\
\hline
\end{tabular}

*denotes statistical analysis was performed by Chi-squared test, and for other variables logistic regression test (single variable analysis) was used 
analysis using Chi-squared and/or logistic regression tests showed no significant difference between the presence of anti- $T$. gondii antibodies (IgG) and demographic and clinical characteristics amongst the patients.

Table 2 shows the prevalence of latent toxoplasmosis, gender, age and IgG titer in patients with COVID-19 and COVID-19 negative subjects. IgG antibodies against this parasite were detected in 72/91 (79.1\%) COVID-19 cases and 96/123 (78.0\%) healthy individuals. The results of the single variable analysis showed that latent toxoplasmosis was not more prevalent in patients with COVID-19 $(\mathrm{OR}=1.1$; 95\% CI, 0.55-2.07, $P<0.85)$. The median and interquartile range (IQR) of IgG titer in patients were (97.3 [31.0-133.5]) and in control groups were (34.4 [13.0-144.5]), indicating no significant difference between the two groups $(P=0.10)$. The median and interquartile range (IQR) of age amongst case and control groups were (41 [IQR: 34-47]) and (30 [IQR: 29-42]), respectively $(P<0.0001)$.

\section{Discussion}

A strong negative covariation between toxoplasmosis and COVID-19 was reported by a study (Jankowiak et al. 2020). The present study found that $84 \%$ of the patients with COVID-19 were positive for anti-T. gondii antibodies (IgG). Previous reports depicted lower levels of $T$. gondii seroprevalence in the north of Iran (Bayani et al. 2013; Kalantari et al. 2018; Rostami et al. 2016). An important associated factor in the epidemiology of toxoplasmosis is age, and several studies demonstrated that the seroprevalence rate of toxoplasmosis increases with age and the peak level was observed in cases older than 50 years (Nasiru Wana et al. 2020; Kalantari et al. 2015). The mean age of the patients with COVID-19 ( $60 \pm 17.6$ years) was higher than the abovementioned studies.
Furthermore, this work showed that the seropositivity rate of latent toxoplasmosis was not significantly increased by age. Also, no significant association between gender and the seroprevalence of latent toxoplasmosis was observed. These results are not in agreement with previous studies reporting a statistically significant association between Toxoplasma seropositivity and age, and gender (Achaw et al. 2019). The possible explanation for this variation is differences in type of study population and study region.

In the present study, the prevalence of toxoplasmosis was not significantly higher in severe cases, which may have confirmed no relationship between SARS-CoV-2 and T. gondii infection.

Moreover, a non-matched case-control study was performed in the second step of the current work which found that the seroprevalence rate for anti- $T$. gondii $\operatorname{IgG}$ antibodies was not significantly different between case and control groups (79.1\% vs. $78.0 \%)$. This finding was not supported by Sharaf-El-Deen and colleagues study which demonstrated the prevalence of toxoplasmosis was significantly higher in patient with COVID-19 than healthy individuals. Their findings also showed the number of Toxoplasma positive cases was statistically significant higher in the severe patient group compared with the moderate patient group (Sharaf-El-Deen et al. 2021). The main possible reasons for this controversy are differences in type of study region and endemicity of toxoplasmosis (Abbas et al. 2020). Toxoplasmosis is an endemic disease in the studied area, and about $60 \%$ of people above 30 years old become exposed to this infection (Rostami et al. 2016; Kalantari et al. 2018).

Our findings demonstrated that the mean, median and IQRs of age in the patient group were significantly higher than in the control group $(P<0.0001)$. According to these results, age was not a matched feature in our study groups, which may have affected the results obtained from the present study. Also, the samples were not collected from the
Table 2 T. gondii exposure amongst patients with COVID19 and subjects without COVID-19 according to age, gender and $\mathrm{IgG}$ titer

\begin{tabular}{|c|c|c|c|c|}
\hline Variable & $\begin{array}{l}\text { Case (91) } \\
N(\%)\end{array}$ & $\begin{array}{l}\text { Control (123) } \\
N(\%)\end{array}$ & OR (CI95\%) & $P$ value \\
\hline \multicolumn{5}{|l|}{ T. gondii exposure } \\
\hline Yes & $72(79.1)$ & $96(78.0)$ & $1.1(0.55-2.07)$ & 0.85 \\
\hline No & $19(20.9)$ & $27(22.0)$ & & \\
\hline \multicolumn{5}{|l|}{ Gender } \\
\hline Female & $48(52.7)$ & $63(51.2)$ & $1.1(0.62-1.83)$ & 0.83 \\
\hline Male & $43(47.3)$ & $60(48.8)$ & & \\
\hline \multicolumn{5}{|l|}{ Age (years) } \\
\hline$<30$ & $18(19.8)$ & $64(52.0)$ & $0.2(0.11-0.39)$ & 0.00 \\
\hline$>30$ & $73(80.2)$ & $55(48.0)$ & & \\
\hline Median and IQRs of age (years) & $41(34-47)$ & $30(29-42)$ & - & 0.00 \\
\hline Median and IQRs of IgG titer & $97.3(31.0-133.5)$ & $34.4(13.0-144.5)$ & - & 0.10 \\
\hline
\end{tabular}


SARS-CoV-2 patients and healthy individuals in the same period which caused limitations in the current work.

\section{Conclusion}

In summary, the present study is a preliminary work that investigated the prevalence of chronic $T$. gondii infection in SARS-CoV-2 patients. The findings demonstrated that latent Toxoplasma infection is prevalent amongst SARSCoV-2 patients. Furthermore, no association was observed between COVID-19 and latent toxoplasmosis. Our results also suggest that further studies with larger sample sizes and with case-control design should be performed to establish any association between $T$. gondii and SARS-CoV-2.

Acknowledgements We are grateful to Shayesteh Shahdeen for the utmost assistance and Taraneh Ghaffari for proofreading the manuscript.

Author contribution NK and SG developed the study, performed data analysis and drafted the manuscript. MB performed medical examination, and all participants were under her supervision. SG drafted the manuscript. TGF edited the manuscript. FJ and MAD collect data and performed experiments. We confirm that all authors have seen and agree with the contents of the manuscript, and there is no financial interest to report.

Funding This work was funded by the Babol University of Medical Sciences with grant number 9910334 .

Data availability The datasets which are used and/or analysed for the current manuscript are available from the corresponding author on reasonable request.

\section{Declarations}

Ethics approval The purpose of the current study was described to the patients, and they gave their informed consent. This work was permitted by the ethical committee of Babol University of Medical Sciences, Babol, Iran (IR.MUBABOL.REC. 1399, 264).

Consent for publication Not applicable.

Competing interests We declare no competing interests.

\section{References}

Abbas I, Villena I, Dubey J (2020) A review on toxoplasmosis in humans and animals from Egypt. Parasitol 147:135-159. https://doi.org/10. 1017/S0031182019001367

Achaw B, Tesfa H, Zeleke AJ, Worku L, Addisu A, Yigzaw N, Tegegne Y (2019) Sero-prevalence of Toxoplasma gondii and associated risk factors among psychiatric outpatients attending University of Gondar Hospital, Northwest Ethiopia. BMC Infect Dis 19:1-8. https://doi.org/10.1186/s12879-019-4234-6

Bayani M, Mostafazadeh A, Oliaee F, Kalantari N (2013) The prevalence of Toxoplasma gondii in hemodialysis patients. Iran Red Crescent Med 15. https://doi.org/10.5812/ircmj.522511
El-Sayed A, Abdel-Daim MM, Kamel M (2021a) Causes of respiratory failure in COVID-19 patients. Environ Sci Pollut Res 28:2882528830. https://doi.org/10.1007/s11356-021-14200-1

El-Sayed A, Kamel M (2021) Coronaviruses in humans and animals: the role of bats in viral evolution. Environ Sci Pollut Res 28:4044540459. https://doi.org/10.1007/s11356-021-12553-1

El-Sayed A, Aleya L, Kamel M (2021b) COVID-19: a new emerging respiratory disease from the neurological perspective. Environ Sci Pollut Res 28:19589-19600. https://doi.org/10.1007/ s11356-021-12969-9

Hassan SA, Sheikh FN, Jamal S, Ezeh JK, Akhtar A(2020) Coronavirus (COVID-19): a review of clinical features, diagnosis, and treatment. Cureus 12. https://doi.org/10.7759/cureus.7355

Jankowiak $€$, Rozsa L, Tryjanowski P, Moller AP (2020) A negative covariation between toxoplasmosis and CoVID-19 with alternative interpretations. Sci Rep 10. https://doi.org/10.1038/s41598-020-69351-x

Kalantari N, Ghaffari S, Bayani M, Elmi MM, Moslemi D, Nikbakhsh N, Ghavipanjeh F (2015) Preliminary study on association between toxoplasmosis and breast cancer in Iran. Asian Pac J Trop Biomed 5:44-47. https://doi.org/10.1016/S2221-1691(15)30169-6

Kalantari N, Sheikhansari MR, Ghaffari S, Alipour J, Gorgani-Firouzjaee T, Tamadoni A, Bayani M (2018) Seroprevalence and molecular detection of Toxoplasma gondii in young healthy blood donors in Northern Iran. Trop Biomed 35:1017-1027

Mackenzie JS, Smith DW (2020) COVID-19: a novel zoonotic disease caused by a coronavirus from China: what we know and what we don't. Microbiol Aust 41:45-50. https://doi.org/10.1177/1010539520931326

NasiruWana M, MohdMoklas MA, Watanabe M, Nordin N, ZasmyUnyah N, AlhassanAbdullahi S, Ahmad IssaAlapid A, Mustapha T, Basir R (2020) A review on the prevalence of toxoplasma gondii in humans and animals reported in Malaysia from 2008-2018. Int J Environ Res Public Health 17:4809. https://doi.org/10.3390/ijerph17134809

Mohanty SK, Satapathy A, Naidu MM, Mukhopadhyay S, Sharma S, Barton LM, Stroberg E, Duval EJ, Pradhan D, Tzankov A, Parwani AV (2020). Severe acute respiratory syndrome coronavirus-2 (SARS-CoV-2) and coronavirus disease 19 (COVID-19)-anatomic pathology perspective on current knowledge. Diag pathol15:1-7. https://doi.org/10.1186/s13000-020-01017-8

Roe K (2021) The symptoms and clinical manifestations observed in COVID-19 patients/long COVID-19 symptoms that parallel Toxoplasma gondii infections. J Neuroimmune Pharmacol 16:513-516. https://doi.org/10.1007/s11481-021-09997-0

Rostami A, Seyyedtabaei SJ, Aghamolaie S, Behniafar H, Lasjerdi Z, Abdolrasouli A, Mehravar S, Alvarado-Esquivel C (2016) Seroprevalence and risk factors associated with Toxoplasma gondii infection among rural communities in Northern Iran. Rev Ins Med Trop Sao Paulo 58:70. https://doi.org/10.1590/S1678-9946201658070

Sala G, Miyakawa T (2020) Association of BCG vaccination policy with prevalence and mortality of COVID-19. Medrxiv. https://doi.org/10. 1101/2020.03.30.20048165

Sharaf-El-Deen SA, Shalan FH, Agha MA, Brakat RM (2021) Toxoplasma gondii as a possible risk factor for COVID-19 severity: a case-control study. Egypt J Med Microbiol 30: 125-132. https://doi. org/10.51429/EJMM30217

Sharma A, Tiwari S, Deb MK, Marty JL (2020) Severe acute respiratory syndrome coronavirus 2 (SARS-CoV-2): a global pandemic and treatment strategies. Int J Antimicrob Agents 56:106054. https:// doi.org/10.1016/j.ijantimicag.2020.106054

Publisher's note Springer Nature remains neutral with regard to jurisdictional claims in published maps and institutional affiliations. 